\title{
„March for Sozialgeographie“? Rechtspopulismus als Zumutung und die regressive Moderne als Herausforderung der Humangeographie
}

\author{
Jürgen Oßenbrügge \\ Institut für Geographie/CEN, Universität Hamburg, 20146 Hamburg, Deutschland \\ Correspondence: Jürgen Oßenbrügge (juergen.ossenbruegge@uni-hamburg.de)
}

Received: 31 March 2018 - Revised: 31 August 2018 - Accepted: 6 October 2018 - Published: 8 November 2018

\begin{abstract}
Kurzfassung. The paper outlines an approach to right wing populism in recent years not only in critizing the use of so called alternative facts but using the concept of a regressive modernisation as a debate which includes populist movements into a broader social theory and diagnosis of western societies. The evolution of regressive modernisation reveals both, the success of a neoliberal globalization and a rise of marginality and perceived dangers of a downward mobility. These findings are used in order to explain certain transformations of academic fields in general and social geography in particular. It is argued that the success of postmodern geographies may be seen as an overcoming of hegemonic discourses of positivism as well as of marxism, but was and is unable to counteract geographies of recent right wing populism. Even the positions of the „march for science“ which has been a major initiative to fight for academic integrity are seen as not being sufficient to rebuilt an ,antipopulist“ social geography. This situation leads to some suggestions and recommendation for further work in this field.
\end{abstract}

\section{Einleitung}

Die Gegenwart ist sicherlich nicht arm an Krisen. Zu nennen sind die bereits länger währenden Wirkungen der Finanzund Wirtschaftskrise, die geopolitischen Krisen und Konflikte, die Eurokrise und das mögliche Auseinanderfallen der EU durch den Brexit, die aktuellen Wahlergebnisse mit den Erfolgen des Rechtspopulismus, die mit der Wahl Donald Trumps einen nicht für möglich gehaltenen Höhepunkt erfahren haben. Aber auch der Diskurs über Unsicherheit, angefacht durch internationalen Terror und Übergriffe durch und gegen Migranten, Abstiegsängste und negative Zukunftserwartungen hat eine Atmosphäre erzeugt, die Bezeichnungen wie Vielfachkrise oder multiple Krise gerechtfertigt erscheinen lässt (Demirovic et al., 2011).

Krisensymptome und gesellschaftliche Unsicherheiten werden von der gesellschaftspolitischen Linken häufig diskursiv vorangetrieben, um beispielsweise die soziale Ungleichheit zu kritisieren, die von Konservativen stillschweigend akzeptiert oder die im neoliberalen Diskurs sogar als Leistungsmotor empfohlen wird. Die linke Kritik an der Ungleichheit und an den von politischen Gegnern vollzogenen
Verbiegungen der sozialen Frage war bisher allerdings politisch nicht sonderlich erfolgreich und gegenwärtig ist eher das Gegenteil der Fall. Rechten Bewegungen ist es nicht nur gelungen, bestehende Ungleichheiten für ihre Unterstützung zu mobilisieren, sondern auch noch populistische Akzente in Form von Schuldzuweisungen, Verachtung und Aggressivität gegenüber Andersdenkenden, Andersgläubigen oder Andersaussehenden hinzuzufügen. Mit der Unterscheidung zwischen dem ,wir“ versus die ,anderen“ und dem ,gemeinen Volk" versus die ,abgehobenen Eliten“"werden Betroffene und Täter diskursiv hergestellt und in populistischen Agitationsformen verfestigt. Sicherlich sind derartige Praktiken und Prozesse, wie der des „Othering"1 , auch in geographi-

\footnotetext{
${ }^{1}$ Dieser Begriff ist stark durch Spivak geprägt worden, um Machtverhältnisse herauszuarbeiten, die sich auf anerkanntes Wissen und Technologie stützen können und gleichzeitig die „Ohnmächtigen" als pathologisch und moralisch minderwertige Andere charakterisieren. In seiner Auseinandersetzung mit Spivaks Ansatz definiert Jensen (2011:65) „Othering“ als ,discursive processes by which powerful groups, who may or may not make up a numerical majority, define subordinate groups into existence in a reductionist way which ascribe problematic and/or inferior characteristics
} 
schen Debatten nicht neu, erinnert sei beispielsweise an die Diskussion über Huntingtons Kampf der Kulturen (Kreutzmann und Reuber, 2002), aber derzeit wieder in beunruhigender Weise allgegenwärtig. Damit kommen in den aktuellen Auseinandersetzungen mehrere Dimensionen zum Vorschein: Zu nennen sind Ungleichheit als strukturelle Dimension sozioökonomischer Differenzierung in vertikaler Richtung und das Othering als diskursive Konstruktion moralischer Überlegenheit und kultureller Hegemonie in horizontaler Richtung. Diese werden durch eine populistische Rahmung in einer sich antielitär und kommunitaristisch inszenierenden politischen Bewegung verbunden.

Vor diesem Hintergrund versteht sich der Beitrag als Diskussionsanregung, um die fachwissenschaftliche Geographie zu positionieren und besonders die Sozialgeographie als dafür wichtiges Diskussionsfeld herauszustellen. Seine Gegenstände sind damit die Entstehung, die Artikulationsformen und die Wirkungen rechtspopulistischer Diskurse, die auch dahingehend hinterfragt werden, ob sie eine mögliche Folge der Wissenschaftspraxis nach dem ,cultural turn“ sind. Weiterhin wird der „March for Science“ als eine neue Gegenbewegung aus der Wissenschaft verstanden, die eine postfaktisch ausgerichtete Medien- und daran angelehnte Wissenschaftspolitik ablehnt und sich dem Rechtspopulismus stellt. Sie dient als Bezugspunkt für entsprechende Aufgaben der Humangeographie unter besonderer Berücksichtigung der damit eingeschlossenen und möglicherweise auch ausgeschlossenen Verständnisse von Wissenschaft. Daraus abgeleitete Empfehlungen werden hier als „March für Sozialgeographie" betitelt.

Um diese Zusammenhänge zu verdeutlichen, geht der Beitrag von vier Thesen aus: Als erstes soll das Aufkommen des Rechtspopulismus verstanden werden als Ausdruck gesellschaftlicher Verhältnisse, in denen kollektive Bindungen verloren gegangen sind und Gruppen entstanden sind, die sich politisch nicht repräsentiert fühlen. Ursache dafür ist der Siegeszug des progressiven Neoliberalismus, der Positionen einer eher marktradikalen Globalisierung mit denen der sozialen Bewegungen zumindest in Teilen verbindet. Diese von Nancy Fraser (2017) vorgetragene These mit Bezug zur Situation in den USA wird hier unter Bezugnahme der Kategorie der regressiven Moderne von Oliver Nachtwey (2016) auf deutsche Verhältnisse übertragen.

Die zweite These bezieht sich auf die Übertragung der Kategorie der „Künstlerkritik“, die von Luc Boltanski und Eve Chiapello (2003) in ihrer Arbeit über den ,neuen Geist des Kapitalismus“ entwickelt worden ist. Dort wird sie verwendet, um die Lern- und Transformationsfähigkeit der kapitalistischen Wirtschaft im Anschluss an die Fordismuskrise zu beschreiben. Im vorliegenden Beitrag wird die Künstlerkritik sowohl in ihrer Beteiligung an der regressiven Mo-

to these subordinate groups. Such discursive processes affirm the legitimacy and superiority of the powerful and condition identity formation among the subordinate". derne als auch als Motor wissenschaftlicher Veränderungen im Kontext der Postmoderne und des Poststrukturalismus verstanden und auf die Entwicklung der Sozialwissenschaften im Allgemeinen und der Sozialgeographie im Besondern seit den 1980er Jahren bezogen. Im Zentrum steht dabei die Aufnahme des postmodernen Denkens, die nicht nur in der Geographie mit der Entwertung konventioneller Wissensbestände, sondern auch mit der Zersetzung des ,,modernen“ Wissensbegriffs einhergegangen ist. Die Frage lautet hier: Ist die Entwicklung der Sozialgeographie (oder Kultur(Sozialgeographie) analog zum Siegeszug des progressiven Neoliberalismus einzuordnen?

Drittens hat die an sich nicht mehr frische Debatte über das Verhältnis zwischen einem modernen und postmodernen Wissensbegriff eine berührende Aktualität, die sich in Inhalten des „March for Science“ 2017 erkennen lassen. Diese Bewegung versteht sich als Kritik an der ,postfaktischen Entwertung" des Wissenschaftsbetriebs besonders in den USA. Dabei geht es allerdings primär um die zu rettende „Science“ (v.a. die Klimawissenschaften), während die interpretative Sozial- und Geisteswissenschaft nicht im Blick ist. Die Verteidigung des wissenschaftlichen Wissensbegriffs nimmt damit ähnliche Züge an wie jene, die Dubiel bereits in den 1970/1980er Jahren als neokonservative Kritik an sozialen Bewegungen und verändertem Wissenschaftsverständnis in den USA ausgemacht hat (Dubiel, 1985). Auch wenn sich die Kritik heute gegen den Rechtspopulismus wendet, bleibt die explizite Würdigung szientistisch geprägter Wissensbegriffe problematisch.

Schließlich und viertens steht die gegenwärtige Diskussion über die Sozialgeographie demnach einer paradoxen Situation gegenüber: Der „March for Science“ bekämpft rechte Momente des Postfaktischen mit einem Wissensbegriff, den dieses Fach als überwunden und als rückschrittlich ansieht. Der gewählte Titel „March for Sozialgeographie“ soll diese Problematik andeuten, wobei der Beitrag abschließend einen einfachen Weg nimmt. Die Debatte über das Postfaktische wird nicht als ein epistemologisches Problem aufgenommen, also nicht als Konflikt unterschiedlicher Begründungen des Wissens. Vielmehr erfolgt ein Plädoyer für die Einheit angesichts einer akzeptierten Vielfalt der Stimmen.

\section{Populismus als Ausdruck der regressiven Moderne}

Spätestens seit der Bundestagswahl 2017 sind auch in Deutschland politische Veränderungen durch die parlamentarische Repräsentation einer Partei deutlich geworden, die neonationalistische, ethnozentrische und gesellschaftlich reaktionäre Positionen und Ziele verfolgt oder zumindest befördert. Was zuvor bereits in anderen europäischen Ländern wie der Schweiz, Polen, Ungarn, Österreich, Frankreich, Italien, Niederlande oder dem Vereinigten Königreich für Unruhe und große Beachtung gesorgt hat, ist mit einer zeitlichen 
Verschiebung, dann aber beschleunigt im Kontext der Flüchtlingsbewegungen im Jahr 2015 und der politischen Auseinandersetzung darüber, auch in Deutschland wirksam geworden und hat besonders rechtspopulistische Bestimmungen des Politischen befördert. In der Geschichte der BRD gab es zwar immer Bewegungen und Parteien, die nationalistische und völkische Positionen deutlich hörbar zum Ausdruck bringen konnten (Holtmann, 2018; Bürk, 2018; Belina, 2013; Oßenbrügge, 1989), jedoch markiert die aktuelle Situation einen generellen Rechtsruck der deutschen Politik und Öffentlichkeit. Um die geläufige Frage, warum es so gekommen ist, zu beantworten, greift dieser Beitrag auf Thesen zurück, die im Kontext der Debatte über die regressive Modernisierung aufgestellt worden sind (Geiselberger, 2017). Zumindest ansatzweise sollen dazu die Bedingungen benannt werden, warum gerade jetzt populistische Agitationen wirkmächtig werden und warum gerade als rechts einzustufende Formen eine hohe Relevanz erhalten. Populismus wird hier nach Mudde und Rivora Kaltwasser $(2017,2018)$ verstanden als (dünne) Ideologie, die nicht auf ein kohärentes Gedankengebäude aufbaut, sondern eklektisch argumentiert. Dennoch lassen sich drei typische Argumentationsrichtungen ausmachen: (a) eine sozioökonomische, die mit Prozessen der ökonomischen Benachteiligung und bzw. oder Prekarisierung verbunden ist, (b) eine soziokulturelle, die auf unterschiedliche kollektive Identitätskonstruktionen aufbaut und die mit dem Hinweis auf „Othering“ bereits angedeutet worden ist sowie (c) eine ,herrschaftskritische“, die besonders die Distanz und den Gegensatz zwischen dem Volkswillen einerseits und den politischen Eliten und der medialen Repräsentation des politisch Verhandelbaren aufnimmt. Besonders der zuletzt genannte Aspekt deutet bereits an, dass Populismus auch immer mit politischen Machtverhältnissen und unterschiedlichen Demokratieverständnissen verbunden ist. Daher besteht auch eine Vielfalt unterschiedlicher und gegensätzlicher Einordnungen populistischer Erscheinungen, die hier allerdings nicht weiter vertieft werden (vgl. u.a. Dubiel, 1985; Priester, 2007; Müller, 2016; Jörke und Nachtwey, 2017).

\subsection{Zum Begriff der regressiven Modernisierung}

Grundsätzlich werden mit Begriffen der Moderne Phasen der gesellschaftlichen Entwicklung beschrieben, die ausgehend von Gedanken der Aufklärung auf Rationalität, Fortschritt und universelle Gültigkeit setzen. Dabei ist die wissenschaftliche Diskussion zum Begriff der Moderne und über die Modernisierungstheorien sehr vielschichtig und strittig (vgl. Peet und Hartwick, 2015; Pollack, 2016). Im Kontext der ,ersten“ Moderne dominierte beispielsweise der wissenschaftlich-technische Fortschrittsglaube, der zusammen mit einer liberal-demokratischen sowie einer nationalund rechtstaatlichen Organisation der Gesellschaft gleichsam automatisch die allgemeinen Lebensbedingungen verbessern würde. Im kritischen Marxismus der Frankfurter Schule kon- kretisierte sich die moderne Haltung dagegen in der Aussage, die Sozialwissenschaft hätte die Aufgabe, den Subjekten die Mittel für ihre Emanzipation von der Entfremdung und Repression zu geben. Strukturalistisch zugespitzt bezog sich diese auf die Befreiung vom Primat der Ökonomie als letztinstanzliche Determination des Sozialen und Basis der Gesellschaft. Die revolutionäre Umwandlung der Gesellschaft wurde dementsprechend das Ziel ,wissenschaftlich“ begründeter Politikansätze der Linken (Marchart, 2013).

Auch wenn die politischen Auseinandersetzungen in der frühen BRD nicht oder nur sehr punktuell diese Reichweite aufgezeigt haben, bestand im politischen Feld zumindest ein konsensueller Schnittpunkt darin, den technischindustriellen Wohlfahrtsstaat, der mit der Chancengleichheit und der sogenannten Aufstiegsgesellschaft zusammengedacht wurde, kontinuierlich auszubauen. Diese häufig auch als fordistisch bezeichnete Phase deutscher Nachkriegsgeschichte ist in den 1970er und 1980er Jahren sowohl in eine wirtschaftliche Krisensituation, die durch das Einsetzen der Massenarbeitslosigkeit charakterisiert werden kann, als auch in eine legitimatorische Krise geraten, an deren Entstehen die Studentenbewegung und später die sozialökologische Bewegung großen Anteil hatte.

Über die Formen und Richtungen, die sich im Postfordismus entwickeln würden oder inzwischen sichtbar geworden sind, gibt es seit Langem eine breite Debatte (Amin, 1994). In der Geographie ist dabei die Auseinandersetzung mit dem Neoliberalismus prägend geworden und Stichworte wie Deregulierung, Globalisierung, Privatisierung, Finanzialisierung veranschaulichen die Bandbreite. Die hier favorisierte Diagnose der regressiven Modernisierung geht von einer bestimmten Interpretation der postfordistischen Transformation aus, die am Beispiel des Befundes der „Abstiegsgesellschaft“" von Nachtwey illustriert werden soll: „Während in der sozialen Moderne $(\approx$ erste Moderne, d. Verf.) der Ausbau der politischen, wirtschaftlichen und sozialen Bürgerrechte seinen Höhepunkt erreichte, wurden diese [...] durch die Kräfte der regressiven Modernisierung ausgehöhlt - und damit zu einem Element der Abstiegsgesellschaft. [...] Die politische Staatsbürgerschaft im Sinne der individuellen Gleichheit schreitet voran. Man könnte sagen, horizontal - zwischen Geschlechtern, Menschen mit unterschiedlichen sexuellen Orientierungen und in bestimmten Bereichen sogar zwischen den Ethnien - wurde die Gesellschaft gleichberechtigter und inklusiver. Zeitgleich wuchs indes der illiberale Umgang mit den Empfängern von Sozialleistungen. Die politische Gleichberechtigung geht vertikal mit mehr Ungleichheit und mitunter mit gestiegener Diskriminierung einher. Die sozialen und wirtschaftlichen Bürgerrechte sind geschrumpft" (Nachtwey, 2016:115).

Regressive Modernisierung spricht damit eine neuartige Form gesellschaftlicher Transformation in sozioökonomischer und soziokultureller Richtung an. Gemeint ist das $\mathrm{Zu}$ sammenspiel wirtschaftlicher Restrukturierung über die neoliberal gesteuerte Globalisierung, den Ausbau globaler Pro- 
duktionssysteme sowie den Bedeutungsgewinn der Finanzialisierung und der Digitalisierung. Diese konnte sich aber nur durchsetzen durch die gleichzeitige und positiv besetzte Stärkung der Subjektebene nicht nur im neoliberalen Sinne des Konsumenten und Arbeitskraftunternehmers, sondern auch in Rechtsansprüchen und Wertschätzung der Diversität und Kreativität. In der Stadtforschung markieren Richard Floridas Arbeiten zur kreativen Klasse und kreativen Stadt geradezu ikonenhaft die Ausstrahlung der kombinierten Wirkung dieser beiden Strukturmerkmale.

Eine konzeptionell richtungsweisende Interpretation dieses Formenwandels vom Fordismus zum Postfordismus haben Boltansky und Chiapello (2003) mit ihrer Untersuchung zum neuen Geist des Kapitalismus vorgelegt. Danach war die intellektuelle Kritik am fordistischen Kapitalismus weniger durch sozialpolitische Motive geprägt, sondern durch etwas, was sie Künstlerkritik nennen. Letztere richtet sich gegen Entfremdung beispielsweise in Form tayloristischer Arbeitsbeziehungen, aber auch gegen hierarchische Organisationsformen und die geringe Selbstbestimmung über Arbeitszeit und Ort. Künstlerkritik hat sich auch gegen den umfassenden Staat mit seiner bürokratischen Kontrolle und der ,formierten“ Gesellschaft artikuliert. Der deutlichste Ausdruck dieser Kritik zeigte sich wohl in den Positionen der sogenannten Alternativbewegung der 1970er und 1980er Jahre (vgl. z.B. Berger et al., 1982). Im Übergang zum Postfordismus floss die Künstlerkritik nicht nur in neoliberale Unternehmensstrategien ein, wobei nach Boltansky/Chiapello den Unternehmensberatungen und der Managementliteratur eine wichtige Rolle zukam, sondern bildete auch den Rahmen für eine weitergehende gesellschaftliche Modernisierung besonders der gesellschaftlichen Gruppen, die wirtschaftliche und soziale Vorteile aus der neoliberalen Transformation erzielen konnten. In der öffentlichen und parteipolitischen Debatte verweist die Diskussion über die ,neue Mitte“ in der deutschen Sozialdemokratie oder über den ,Third Way“ in Großbritannien auf die Bedeutung dieser Verschiebungen am Beispiel der Überlegungen zur Frage, wer nach der Erosion der Industriearbeiterschaft für eine Massenunterstützung der sozialdemokratischen Parteien in Wahlen sorgen könnte.

Der Begriff der regressiven Moderne betont demnach besonders zwei politisch bedeutsame gesellschaftliche Kontexte: Auf der einen Seite befinden sich die Modernisierungsgewinner. $\mathrm{Zu}$ ihnen gehören die wirtschaftlichen Profiteure der neoliberalen Transformation und Repräsentanten des globalen Konsumkapitalismus, aber auch die Gewinner des Abbaus horizontaler Ungleichheiten und der Aufwertung der Selbstentfaltung und postmaterialistischer Wertvorstellungen. Die Zusammenfassung beider Gruppen ist in der Bezeichnung progressiver Neoliberalismus erfolgt, die Nancy Fraser (2017) in Bezug auf die Situation in den USA gewählt hat und die hier besonders treffend erscheint (andere gängige Bezeichnungen sind z.B. Kosmopolitismus, Merkel, 2017). Auf der anderen Seite stehen die Kritiker dieser selektiven Modernisierung und die Globalisierungsver- lierer, die sich durch die sich entgrenzende Ökonomie als gefährdet und abgehängt wahrnehmen und sich sozialkulturell in Folge der Infragestellung und Dekonstruktion von tradierten Identitätsbildern als ausgegrenzt ansehen. Die regressive Moderne erzeugt somit soziale Milieus, deren politische Subjektivität durch Kränkungserfahrungen, Verlustund Statusängste oder Zumutungen bei den Sozialleistungen geprägt sind. Während solche Wahrnehmungen und Erfahrungen als Empfinden verletzter Gerechtigkeit, als Ausbleiben sozialer Anerkennung und als vermeintlicher Verlust kultureller Identität in politisch stabilen und kulturell hochintegrierten Gesellschaften ,weitgehend eingebunden in die von politischen und kulturellen Eliten vordefinierten Legitimationsangebote" bleiben (Dubiel, 1985:648), bilden sie heute die Ressource rechtspopulistischer Agitation. Verstärkend wirkt eine Sachzwangs- und Verwaltungspolitik, die auf elitär-technokratisch erscheinenden Entscheidungsprozessen beruht, sich über Globalisierungsanforderungen legitimiert, gleichzeitig aber den demokratischen Streit über politische Fragen sinnentleert. Skeptische Haltungen gegenüber liberaldemokratischen Organisationsformen nehmen auf allen Ebenen zu, die zu zahlreichen Diagnosen der Postdemokratie geführt haben (vgl. Crouch, 2008, aber auch Blühdorn, 2013). Die Bezeichnungen „Wutbürger“ oder ,Zornpolitik“(Jensen, 2017) stehen für die weitgehende Entfremdung von den als legitim erachteten demokratischen Verfahren über repräsentativ organisierte Parlamente und sind ein Ausdruck für das Entstehen sozialer Potentiale, die in die herkömmlichen politischen Willensbildungs- und Entscheidungsprozesse nicht eingebunden sind. Somit verweist der Begriff der regressiven Modernisierung auch auf ein erhebliches und sicherlich auch lange unterschätztes Demokratieproblem. Gleichzeitig wächst damit ein weiterer populistisch nutzbarer Kontext, der als Responsivität bezeichnet wird. Bewegungen, Parteien und Politiker erhalten dann starke Unterstützung, wenn sie sich als unmittelbares Sprachrohr und konsequente Vertreter des ,Volkswillen“ darstellen. Im Kontext der postdemokratischen Gesellschaftsdiagnose handelt es sich dabei aber weniger um eine Reaktivierung liberal-demokratischer Entscheidungsformen, sondern um den Ausdruck der Oppositionshaltung gegen die politische Elite und die Durchsetzung eines sogenannten Mehrheitswillens auch gegen Minderheitsrechte (Mudde und Rivora Kaltwasser, 2018; Steiner und Landwehr, 2018).

\subsection{Rechtspopulismus als Ausdruck der regressiven Moderne}

Die Befunde zur regressiven Moderne lassen in Hinblick auf die aktuelle Situation politische Schlussfolgerungen zu, die unterschiedliche Kriseninterpretationen implizieren. $\mathrm{Zu}$ nächst liegt es nahe, das Erstarken des Rechtspopulismus auf die erfolgreiche politische Mobilisierung der Modernisierungsverlierer durch PEGIDA, AfD und andere zu interpretieren. Danach hätten diejenigen, die im Kontext kriti- 
scher Sozialforschung und Analysen zunehmender Deklassierungen in Kategorien wie Underclass oder Prekariat gefasst worden sind, im rechten Diskurs ein neues Zuhause gefunden. Jedoch sind die bisherigen empirischen Befunde uneinheitlich und lassen unmittelbare Beziehungen zwischen sozialer Benachteiligung und Unterstützung rechtspopulistischer Strömungen als fragwürdig erscheinen (Mudde und Rivora Kaltwasser 2018:7). Bedeutender erscheinen die auf eine unsichere Zukunft gerichteten Abstiegsängste solcher Bevölkerungsgruppen, die zwar ökonomisch nicht der Unterschicht zuzuordnen sind, aber verminderte oder keine Aufstiegsperspektiven mehr sehen und daher ihren Statuserhalt in Frage stellen (Koppetsch, 2017). Erste wahlgeographische Analysen der AFD-Unterstützung in der deutschen Bundestagswahl 2017 zeigen dazu folgendes Bild: „Der Zuspruch für die AfD war in (den) Wahlkreisen relativ hoch, in denen überdurchschnittlich viele Handwerksunternehmen angesiedelt, überproportional viele Erwerbstätige im verarbeitenden Gewerbe beschäftigt sind, sowie - das gilt eher für die westdeutschen Wahlkreise - die verfügbaren Haushaltseinkommen unter dem Bundesdurchschnitt liegen. Überdies wird häufig in solchen Wahlkreisen für die AfD gestimmt, in denen überdurchschnittlich viele Ältere leben“" (Franz et al., 2018). Die sich zeigende politische Geographie hat Hillje (2018) über tiefergehende qualitative Interviews in solchen städtischen und ländlichen Gebieten, die als strukturschwach und affin für rechtspopulistische Rhetorik gelten, als „Räume der politischen Verlassenheit“ bezeichnet. Angesprochen wird eine Mischung aus sozialer Benachteiligung, infrastrukturellen Defiziten und Vertrauensverlust gegenüber einer Verbesserung der Alltagssituation durch etablierte politische Repräsentanten.

Durch Globalisierung erzeugte Statusängste, Abstiegserfahrung und politisch-kulturelle Entfremdung sind gut anschlussfähig an ethnonationalistische Konzepte. So hat inzwischen die soziale Frage in Form einer ,exkludierenden Solidarität" (Bieling, 2018) Eingang in die rechtspopulistische (und rechtsradikale) Rhetorik gefunden. ${ }^{2}$ Jedoch ist der Rechtspopulismus in Deutschland und vielen europäischen Ländern nicht nur und auch nicht primär sozioökonomisch ausgerichtet, sondern wendet sich vor allem gegen Zuwanderung, gegen islamische Religionen und Diskurse über Multikulturalismus. Er verhält sich zudem respektlos und aggressiv gegenüber Menschen, die nicht dem heteronormativen Geschlechtermodell folgen. In der Betonung und Rückgewinnung der ethnonationalistisch definierten Kontrolle ist rechter Populismus nicht nur islamophob, sondern in mehr-

\footnotetext{
${ }^{2}$ Ein markantes Beispiel zitiert Grumke (2016:141): ,Die einzige wirksame Waffe gegen das internationale Kapital ist das grenzensetzende, volk- und heimatbezogene bodenständige Gegenprinzip des Nationalismus. Gegen die kapitalistische Entwürdigung des Menschen kämpft der Nationalismus mit seiner sozialen Ordnungsidee der Volksgemeinschaft".
}

facher Hinsicht eindeutig repressiv und enthält zahlreiche autoritäre Reflexe. ${ }^{3}$

Die Bedeutungszunahme des rechten Populismus lässt sich damit als Produkt und als Reaktion auf den Strukturwandel interpretieren, durch den der fordistische Kapitalismus durch Einzug neoliberaler Dominanz und zunehmender Gleichberechtigung grundlegend transformiert worden ist. „Die Hochphase der Globalisierung begünstigte [...] die Etablierung einer kosmopolitisch orientierten Bewusstseinsindustrie, die ihre Wachstumschancen darin sah, den Expansionsdrang kapitalistischer Märkte mit den libertären Werten der sozialen Revolution der sechziger und siebziger Jahre sowie deren utopischen Versprechen menschlicher Befreiung aufzuladen. Dabei verschmolz die technokratische pensée unique des Neoliberalismus mit dem moralischen juste milieu einer internationalistischen Diskursgemeinschaft.“ (Streeck, 2017:259). Neben Deregulierung und Globalisierung, Privatisierung und Beförderung marktförmiger Konkurrenzverhältnisse sind gleichzeitig solche Komponenten umgesetzt und systemimmanent integriert worden, die als Künstlerkritik am Fordismus entstanden sind. Auf diese Weise ist eine zugleich ökonomische und kulturelle Hegemonie wirksam geworden, die den gesellschaftlichen Wandel in liberalen Formen geprägt hat. Ihre kollektive Bindungswirkung ist aber begrenzt und größere Bevölkerungsgruppen sind herausgefallen. Dadurch haben sich politisch-kulturelle Freiräume ergeben, die inzwischen rechtspopulistisch besetzt worden sind. Die neuen rechten Bewegungen betonen eine ethno-nationalistische Transformation, die zumeist auf Mythen der Vergangenheit aufbaut und reaktionäre Momente im Sinne autoritärer, patriarchalischer und eigennütziger Positionen aufruft. Die einleitend mit dem „Othering“ beschriebene Haltung dominiert, die Wodak (2018) treffend mit „schamloser Normalisierung“ beschrieben hat.

\section{Die Sozialgeographie in der regressiven Moderne}

Als Ausgangsthese für den folgenden Abschnitt stellt sich die Frage, ob die besprochenen Veränderungen auch durch den Bedeutungsgewinn relativistischer Wissenschaftsbegriffe und durch neue Schwerpunktsetzungen der Forschungsinteressen befördert worden sind. Haben somit auch die Wissenschaft, die Geographie, die Sozialgeographie einen Anteil an der Regression? Im Rückbezug auf den vorhergehenden Abschnitt wäre die These folgendermaßen zu konkretisieren: Ähnlich wie die Künstlerkritik am Fordismus besonders die Arbeitsbeziehungen transformiert hat, ohne allerdings dabei die Kapitallogik ernsthaft zu gefährden, sondern vielmehr dem neoliberalen Strukturwandel einen progressiv erscheinenden Charakter verliehen haben, so ließen sich die Post-

\footnotetext{
${ }^{3}$ Mudde und Rivora Kaltwasser (2018) verweisen aber hier auf den Aspekt, dass es weniger die Zuwanderung als solche, als vielmehr die durch die Eliten geförderte bzw. tolerierte Zuwanderung ist, die das populistische Moment befördert.
} 
Tabelle 1. Der wissenschaftliche Wissensbegriff im modernistischen Verständnis.

1. Wissenschaftliches Wissen bezieht sich auf Fakten, die das Ergebnis eines durch nachvollziehbare Methoden gesteuerten Erkenntnisprozesses sind. Fakten können zu Faktengebäuden, d.h. Theorien und Modellen, zusammengefügt werden.

2. Wissenschaftliche Fakten haben einen Wahrheitsanspruch, der jedoch kritisch reflektiert wird und damit keinen Anspruch auf absolute Gewissheit impliziert.

3. Wissenschaftliche Objektivität bleibt relativ, ist aber nicht beliebig. Die angemessene wissenschaftliche Haltung ist daher die Anerkennung ihrer Erkenntnisse und die skeptische Hinterfragung derselben.

4. Wissenschaft ist empirisch, setzt sich mit beobachtbaren Phänomenen auseinander. Wissenschaftliche Fakten, Theorien und Modelle werden durch empirische Vorgehensweisen bestätigt oder verworfen.

5. Wissenschaft er- und zersetzt in diesem Sinne traditionelle Weltbilder (oder das narrative Wissen) in einer als aufklärerisch gemeinten Richtung.

moderne und andere Postbewegungen als Künstlerkritik der zuvor dominierenden wissenschaftlichen Paradigmen verstehen. Damit liegt auch die Frage nahe, ob sie unsere Kritikfähigkeit an gesellschaftlichen Verhältnissen gesteigert oder möglicherweise geschwächt hat? Letzteres ist immer wieder und auch aktuell beklagt worden. ${ }^{4}$ Als Arbeitshypothese soll hier ein provozierendes Zitat aus einem Artikel dienen, der in der „,Journal of Democracy“ erschienen ist: „Im linken intellektuellen Lager entstand aus der Frustration, postkapitalistische Verhältnisse zu erreichen, und aus Langeweile, sich mit den Banalitäten und dem Materialismus der bestehenden Ordnung zu beschäftigen, eine Suche nach neuen Themen. Sie wandten sich ab von nützlichen Kapitalismusanalysen und betonten stattdessen die Postmoderne, den Multikulturalismus, Feminismus, Postkolonialismus und eine Bandbreite anderer intellektueller Trends, die eher dem Kulturellen als dem Ökonomischen zuzurechnen sind. Diese Trends hatten wenig Relevanz für den Durchschnittsbürger und waren besonders unattraktiv für die Arbeiterklasse. Sie beförderten eher die Fragmentierung der Bürgerschaft statt sie zu vereinen, wodurch der Aufbau progressiver Koalitionen zunehmend schwieriger geworden ist. Als Ergebnis dieser Trends versagte die Linke darin, genügend Energie und strategisches Denken über den Wandel der europäischen Ökonomien und Gesellschaften in den letzten Jahrzehnten und über nötige Interventionen bereitzustellen“ (Berman, 2016:7, Übersetzung d.V.). Nun gehört es gewiss nicht zu den primären Aufgaben der Human- oder der Sozialgeographie, die gesellschaftliche Linke als politische Kraft zu stärken. Aber Berman kritisiert auch die häufig gefeierte Pluralität und Diversität der fachwissenschaftlichen Ansätze, die geradezu stilbildend für die jüngere Entwicklung der Humangeographie gewesen ist, als kontraproduktiv für das Erreichen politisch

\footnotetext{
${ }^{4}$ Vgl. u.a. Koschorke (2018): Die akademische Linke hat sich selbst dekonstruiert. Es ist Zeit, die Begriffe neu zu justieren. NZZ18.04.2018; Koppetsch (2018): Viele Linke machen sich etwas vor. Taz 7.7.2018; Paoli (2017): Die lange Nacht der Metamorphose. Über die Gentrifizierung der Kultur. Berlin.
}

emanzipatorischer Ziele. Die im Zitat ausgedrückte Kritik soll daher hier als Aufforderung verstanden werden, über die gesellschaftliche Relevanz der Humangeographie und ihre Veränderungen in der jüngeren Fachgeschichte nachzudenken. Dieses kann in diesem Beitrag allerdings nur selektiv und holzschnittartig erfolgen und ist eher als Anregung und Diskussionsbemerkung gemeint. Zwei Schritte werden für die Umsetzung vollzogen. Zunächst soll an den Kontext der postmodernen Herausforderung erinnert werden, um Merkmale der Differenz zwischen modernistischen und sich davon abgrenzenden Wissensbegriffen in den Sozialwissenschaften ansatzweise zu bestimmen. Im zweiten Schritt werden daran anschließend einige Fragen und Thesen mit Bezug zur Sozialgeographie in illustrierender, dadurch möglicherweise auch provozierender Absicht angehängt.

\subsection{Postmoderne Transformation der Sozialwissenschaft}

Das moderne Verständnis vom wissenschaftlichen Wissen lässt sich mit einigen bekannten Prinzipien beschreiben, die hier nur deshalb aufgeführt werden sollen, um die Differenz zu postmodernen Auffassungen zu verdeutlichen (Tabelle 1). Sie werden später aber auch noch wichtig, um diejenige Positionsbestimmung gegen das sogenannte Postfaktische zu benennen, die im „March for Science“ artikuliert worden ist. Die modernistische Auffassung des wissenschaftlichen Wissens geht in ihrer Konsequenz von einer Überlegenheit ihrer Vorstellung gegenüber anderen Wissenskonzepten aus, wodurch auch der Anspruch legitimiert werden kann, ein wissenschaftlich angeleiteter gesellschaftlicher Wandel sei fortschrittlich (zur kritischen Einschätzung bereits Beck, 1986). Dieser Anspruch wird von Vertretern der Postmoderne bestritten. Eine der wichtigsten Arbeiten und programmatischen Herausforderungen des modernistischen Wissenschaftsverständnisses hat Jean Francois Lyotard Anfang der 1980er Jahre in seiner Schrift „Das postmoderne Wissen ein Bericht“" vorgelegt, der zwischen einem wissenschaftli- 
chen und einem narrativen Wissensbegriff unterscheidet. Der narrative Wissensbegriff bezieht sich auf ein traditionelles Wissen, Lyotard spricht auch von Meinungen. Dieses Wissen dient der Überlieferung von Gewohnheitsregeln, gibt den Beteiligten eine Möglichkeit zur Selbstvergewisserung und bestimmt damit auch, ,was in der Kultur das Recht hat, gesagt und gemacht zu werden, und da sie selbst einen Teil von ihr ausmachen, werden sie eben dadurch legitimiert" (Lyotard, 1982). Allerdings behauptet Lyotard nicht, dass das Narrativ einerseits und die wissenschaftliche Erkenntnis andererseits dieselbe Berechtigung auf Anerkennung haben, sich also ersetzen können oder beliebig austauschbar seien. Aber er stellt mit seiner postmodernen Kritik Argumente bereit, die zur Dekonstruktion der modernistischen Wissenschaftsauffassung geführt haben. Letztere hätte als emanzipatorische Erzählung mit ihrer Verbindung von Wahrheit und Gerechtigkeit und ihrer fortschrittsoptimistischen Vorstellung von Aufklärung jegliche Glaubwürdigkeit verloren. Die Postmoderne, so das von Lyotard begründete Selbstverständnis, lehrt die Wissenschaft ,die raue Nüchternheit des Realismus“.

In der postmodernen Welt bestehen vielfältige Narrative und Meinungen, die nicht verbunden sein müssen, oder - und wichtiger - nicht mehr miteinander durch eine übergeordnete Instanz, die Vernunft, verbunden werden können. Während einflussreiche Sozialtheoretiker wie Habermas noch auf die kommunikative Vernunft gesetzt haben, die das Potential einer Lösung nicht nur wissenschaftlicher Probleme, sondern auch lebensweltlicher Konflikte mit sich führe, wird dieser Gedankengang in der postmodernen Kritik zu einer hoffnungslos romantischen Haltung.

Man mag das Ende der großen Theorie, das Zweifeln an epistemologischen Metanarrativen oder die als zwanghaft empfundene Entscheidung, sich entweder den Varianten des Szientismus oder denen der Kritischen Theorie und des Marxismus zuordnen zu müssen, sicherlich begrüßen können. Ein Argument, das Berman in dem Zitat aufführt, sticht zumindest im Hinblick auf die deutschsprachige Situation sicherlich nicht: Die Post-Debatte ist keine Reaktion auf die vermeintliche Unveränderbarkeit des Kapitalismus gewesen, sondern sie zielte auf Dogmen und Prinzipien ab, die als Herstellung und Aufrechterhaltung wissenschaftlicher Meinungsführerschaft angesehen wurden. Der Einstieg in die Postmoderne lässt sich daher auch als machtkritische Bewegung ansehen. Entsprechend waren erste sozialgeographische Aufarbeitungen (z.B. Hasse, 1988; Krüger, 1988) eher „Befreiungen“ und markieren Aufbruchstimmungen. Sie sind aber wegen der Möglichkeiten für einen prekären Relativismus auch früh kritisch eingeschätzt worden (Harvey, 1989; Becker, 1996). Aktuell werden postmoderne Auffassungen und die Ablehnung eines aufklärerischen Universalismus des modernen Denkens auch von rechts besetzt. ${ }^{5}$

\footnotetext{
${ }^{5}$ So verzichten Positionspapiere der ,identitären Bewegung“ oder der Herausgeber der Zeitschrift „Sezession“ auf rassistische Argumente, indem sie ein Konzept des monoethnischen Staates pro-
}

Vor diesem Hintergrund gewinnt die gegenwärtige Kritik an der Postmoderne mit der ihr zuschreibbaren ,,Vielfaltseuphorie" (Heisterhagen, 2018:5), auf die sich die Vertreter der identitären Bewegung berufen, an Bedeutung und sollte zur fachwissenschaftlichen Reflektion anregen.

\subsection{Postmoderne Transformation der Sozialgeographie}

Welche Relevanz haben diese Überlegungen für die jüngere Entwicklung der Humangeographie? Die frühe Aufbruchsphase des Faches besonders im angelsächsischem Raum in den 1970er und 1980er Jahren war zunächst wie die Soziologie vom später sogenannten methodologischen Nationalismus geprägt. Anders ausgedrückt bildete der territoriale Nationalstaat (ebenso wie die Klassenstruktur) den Rahmen und Bezugspunkt für Auseinandersetzungen um Erfolge, Probleme und Widersprüche der systemischen, sozialen und symbolischen Integration der Gesellschaft. Um dieses in verkürzter Form zu illustrieren, lassen sich die frühen Jahrgänge solcher Zeitschriften aufrufen, die diese Aufbruchsphase charakterisieren. Ein Beispiel ist die „Antipode“ mit den dort publizierten Beiträgen zur systemischen Integration, den Geographien der Arbeit, der sozialen und räumlichen Arbeitsteilung und den Kritiken am „Spätkapitalismus“ aus ,welfare-“ bzw. ,radical-Perspektive“. Ähnliches gilt für Fragestellungen der sozialen Integration mit Blick auf die Zeitschrift „Society and Space“, in denen am Anfang neben der Debatte über Haupt- und Nebenwidersprüche des Kapitalismus und den Herausforderungen feministischer Kritik ein erweiterter Blick auf Emanzipation und Machtverhältnisse verfolgt wurde. Schließlich ließe sich die Zeitschrift „Political Geography“ als Beispiel für die Auseinandersetzung über symbolische Integrationsformen, politische Regulation und Territorialität hervorheben. Für alle diese Fragen war der territoriale Nationalstaat ein überragender Bezugspunkt, um gesellschafts- und kapitalismuskritische Analysen zu realisieren.

Die postmoderne Sozialgeographie hat diese methodologische Prämisse nicht nur aufgegeben, sondern umstandslos in die Kritik der Containergeographie einbezogen. Damit ist dem Bezug auf den territorialen Nationalstaat und dem methodologischen Nationalismus eine ähnliche Naivität unterstellt worden, die zu Recht an Beispielen wie dem länderkundlichen Schema, dem ökologischen Fehlschluss oder

pagieren und diesen mit Verweisen auf die Geschichte der europäischen Nationalstaatlichkeit legitimieren. Der Ethnopluralismus kritisiert aber auch Zuwanderung von Personen aus anderen Kulturen, da sie zum Verlust von Identität führen würde. Volksgemeinschaft ist somit auch mit (allerdings verkürztem und selektivem) Rückgriff auf die postmoderne Betrachtung von Wissensbegriffen begründbar. In zugespitzter Form: Wie die Künstlerkritik am Fordismus in einem globalen Neoliberalismus aufgegangen ist, kann die postmoderne Kritik am modernistischen Wissenschaftsbegriff und ihren Folgen als ein Steigbügelhalter ,wissenschaftlich untermauerter" Positionen des Rechtspopulismus angesehen werden. 
der Handlungsfähigkeit von Räumen herausgearbeitet worden ist. Mit Ankunft in der Postmoderne, so die These, wird auch in der Sozialgeographie ein markanter Zersetzungsprozess sichtbar, der im deutschsprachigen Raum mit der Etablierung der „Neuen Kulturgeographie“ einen Höhepunkt der Vielfalt, Offenheit und Experimentierfreude erlebt hat.

Eine Kultur- oder Sozialgeographie als Konglomerat von diversen Narrativen mit ex- oder impliziten Raumbezügen kann sicherlich als die konsequente Weiterführung der bereits angedeuteten Aufbruchstimmung im Fach angesehen werden. Neben der im deutschsprachigen Raum besonders mit dem Namen Benno Werlen verbundenen Rahmung der Sozialgeographie, die ein vergleichsweise einheitliches sozialtheoretisches Programm verfolgt, hat sich unter dem Label „,neue Kulturgeographie“ ein bunt besetzter Marktplatz unterschiedlicher Theorie- und Themenangebote entfaltet, der bisher selten in Hinblick seiner gesellschaftspolitischen Relevanz hinterfragt worden ist. Eine für den hier gewählten Einstieg nützliche Ausnahme stellt Lossaus Rückblick auf die ersten Jahre der neuen Kulturgeographie dar (Lossau, 2008), der sich mit den Begriffen Beliebigkeit, Unwissenschaftlichkeit und Weltfremdheit beschäftigt. Während die ersten beiden Begriffe, die sie als mögliche Kritikpunkte an der Neuen Kulturgeographie diskutiert, in der Auseinandersetzung um die Postmoderne von Anfang an präsent gewesen sind (z.B. fehlende machtstrukturelle Perspektive und die ungenügende analytische Tiefenschärfe narrativer Wissensbegriffe), ist der dritte Begriff, der Vorwurf der Weltfremdheit, besonders interessant. Lossau konkretisiert ihn über die Frage, ob ,die Beschäftigung mit intellektualistischen und ,abgehobenen" Theorien in der Neuen Kulturgeographie dazu geführt [... habe], dass die ,ganz normale Welt‘ und die Alltagspraktiken von ,ganz normalen Menschen' aus dem Blick geraten sind" (ebd. 328) und kommt damit den aktuell geäußerten Monita über die sich gesellschaftskritisch gebende Wissenschaft sehr nahe (siehe Fn. 4). Darüber hinaus werden in dem Beitrag von Lossau neben den als berechtigt bezeichneten fachlichen Herausforderungen Ausblicke für eine Weiterentwicklung des ,cultural turns“ in der Humangeographie gegeben. Auch wenn ein entsprechender Review-Artikel aktuell nicht vorliegt, um inzwischen eingetretene Veränderungen zu bilanzieren und dieses im vorliegenden Beitrag nicht realisiert werden kann, ließe sich das Gesamtergebnis vermuten. Es gibt zahlreiche Anzeichen dafür, die skizzierten Herausforderungen als weitgehend überwunden anzusehen. Dafür sprechen beispielsweise so unterschiedliche Aspekte wie der Bedeutungsgewinn praxistheoretischer Ansätze, die Beachtung der „kritischen Geographie“ in der Fachöffentlichkeit oder die Besetzungspraxis humangeographischer Lehrstühle und die Forschungsförderung.

Gleichwohl ist zu konstatieren, dass wir der alten Aufgabe der Wissenschaft, Mittel für die Emanzipation und gegen Repression bereit zu stellen, dadurch nicht viel näher gekommen sind. Gleichzeitig verblüfft die Widerstandskraft des territorialen Paradigmas außerhalb der Humangeographie, das nach zwei Jahrzehnten geographischer Dekonstruktionsversuche derzeit einen neuen ,internationalen“ Nationalismus mit ,völkischem“ Grundton über rechtspopulistische Positionen begründet und rechtfertigt. Zusammen mit der Kritik an Zuwanderung und Multikulturalismus, die als ,überhörte" Stimme des Volkes legitimiert wird, breitet sich derzeit eine politische Geographie völkisch bestimmter Territorialitäten aus, der wir bisher wenig entgegenzusetzen in der Lage sind. So gesehen kann einer weiteren Diagnose von Berman durchaus zugestimmt werden: Die Neuentwicklungen der Kultur-/Sozialgeographie haben wenig Wirksames ergeben, um diesen ,roll-back“ in den Blick zu nehmen und zu verhindern. Ratlosigkeit beschreibt die Situation wohl treffender.

Möglicherweise ist dieses kein besonderes Problem der Sozialgeographie und ihrer Entwicklung in den letzten Jahrzehnten. Vielmehr mögen sich hier allgemeine Tendenzen der Sozialwissenschaft spiegeln, die sich zunehmend ausdifferenziert hat, einen akademischen Diskurs pflegt und dabei eine politisch intervenierende Rolle vermissen lässt. Was ist in einer derartigen Situation zu tun? Sollten wir uns darauf zurück besinnen, in der Wissenschaft ein Mittel nicht nur zum Zweck der Freiheit zu sehen, sondern besonders eines, das der Emanzipation des Menschen dient und ethischpolitischen Zielen der Aufklärung folgt? Sollte sich Wissenschaft wieder primär durch universalistische Prinzipien definieren, um den postfaktischen Zumutungen und rechten Populisten etwas entgegenzusetzen? Unzureichend wäre es sicherlich, sich zurückzulehnen und zu hoffen, der Lauf der Zeit würde einen Bereinigungsprozess erzeugen. Die Wissenschaftsgeschichte ist voller Rückschritte, intellektueller Deformationen und nachhaltiger Irritationen. Die Geschichte der Politischen Geographie in Deutschland ist ein naheliegendes Feld für entsprechende Belege. Die Initiatoren des „March for Science“" haben daher die Zeichen richtig gedeutet und eine öffentliche Mobilisierung erzeugt, die den gegenwärtigen und zukünftigen Wert des wissenschaftlichen Wissens herausstellen soll. Diese Bewegung ist gegenwärtig ein sichtbarer wissenschaftspolitischer Ausdruck, der angesichts der Herausforderungen der regressiven Moderne Gegengewichte ins Spiel bringt. Jedoch, wofür wird mobilisiert? Und ist es eine Positionierung, die für eine Erneuerung der Sozialgeographie taugt?

\section{Vom ,March for Science“ zum ,March for Sozialgeographie"}

Die sicherlich als dreist charakterisierbaren Aussagen der Trump-Administration beispielsweise hinsichtlich der öffentlichen Teilnahme bei der Regierungsübernahme des derzeitigen US-Präsidenten oder in Hinblick auf die Gefahren des Klimawandels haben eine breite Debatte über die Bedeutung sogenannter alternativer Fakten oder das Postfaktische erzeugt. Besonders die Herabsetzung des wissenschaftlichen 
Wissens als ein mögliches Narrativ unter anderen, dem keine besondere Geltung und keine legitimen Wahrheitsansprüche zuzubilligen seien, hat Wissenschaftlerinnen und Wissenschaftler verschiedener Fachrichtungen mobilisiert und seit Anfang 2017 zu zahlreichen Veranstaltungen, Demonstrationen und Kundgebungen geführt. In den Veranstaltungen an deutschen Universitätsstandorten wurden zudem die Unterdrückung und die Einschränkungen der wissenschaftlichen Freiheit besonders in der Türkei oder Indien, aber auch in Ungarn thematisiert und Unterstützungen verfolgter Wissenschaftler diskutiert und angeboten. Erstmals seit vielen Jahren haben damit Akteure der Wissenschaft versucht, auf die öffentliche Meinung gezielt einzuwirken und eine offensive Wissenschaftskommunikation betrieben. Die Beteiligten sprachen auch über „open science“, „citizen science“ oder andere Formen, den Dialog zwischen Wissenschaft und Gesellschaft zu befördern, um auf diese Weise dem Abwertungsversuch rechtspopulistischer Strömungen zu begegnen.

Da hiermit ein Debattenbeginn markiert worden ist, lassen sich der Gehalt und die Bedeutung des „March for Science“ nur vorläufig bewerten. Sicherlich sind Motivationen und Bewegungsformen unstrittig, schwieriger ist die Einschätzung dessen, was als das wissenschaftlich Faktische und damit als Gegengewicht zum Postfaktischen herausgestellt worden ist. ${ }^{6}$ Thesenhaft zugespitzt verweist der „March for Science“ aber eindeutig auf ein modernistisches Konzept: Die Inhalte zielen auf ein Wissenschaftsverständnis ab, das das Postfaktische als „fake“ demaskiert und das anderen Formen des narrativen Wissens überlegen ist. Trotz der praktizierten Offenheit dominiert eine Haltung, die Wissenschaft von gesellschaftlichen Kontexten löst, Prinzipien der Kontingenz auf Falsifikationsmöglichkeiten reduziert und einer Rationalität folgt, die Faktoren wie politische Subjektivität, Ideologie und grundlegende Konflikte ausklammert.

Es wird eine paradoxe Situation sichtbar: die Inhalte des „March for Science“, die eine breite Unterstützung erfahren haben, schließen die Sozialgeographie nach ihrer postmodernen Transformation nicht oder nur teilweise ein. Mehr noch: Einer Sozialgeographie, die dem Ideal der im March for Science ausgedrückten Haltung entsprechen würde, ist bereits im Sammelband Kultur-/Sozialgeographie von Peter Sedlacek und anderen Anfang der 1980er Jahre mit Hinweis auf eine aus damaliger Sicht nötige normative Orientierung ein Abgesang gewidmet worden (Sedlacek, 1982). Was aber wären richtungsweisende Inhalte eines „March for Sozialgeographie“, der sowohl die postfaktischen Herausforderungen annimmt und ihnen begegnet, gleichzeitig aber auch die verschiedenen Neuentwicklungen in die fachwissenschaftliche Diskussion einpflegt? Zu wenig erscheint der mögliche

\footnotetext{
${ }^{6}$ Die folgenden zugespitzten Aspekte beziehen sich auf die Göttinger Demonstration und Kundgebung zum „March for Science“ am 22. April 2017, die in den Ansprachen, aber auch bei den Beteiligten, soweit sie ihre Positionen mit Plakaten und Bannern zeigten, offensichtlich geworden ist.
}

direkte Rückgriff auf Lyotard mit dem Verweis, bei dieser Frage würde ein agonales Sprachspiel sichtbar, eine Kontroverse zwischen verschiedenen Narrativen, die jetzt und in Zukunft auszutragen wären. Wissenschaft müsse daher neurechte Populismen und auch andere Fassungen des Postfaktischen lediglich dekonstruieren und damit ihre intellektuelle Ärmlichkeit belegen. Zielführender wäre eine Reflektion über diese Frage in der Wiederaufnahme des bekannten Diktums von Habermas: die Einheit der Vernunft sei allein in der Vielfalt ihrer Stimmen vernehmbar. Diese gedachte Überbrückung moderner und postmoderner Begriffe wäre ein erster Schritt und unabhängig der Einheitsfrage für die Sozialgeographie bereits sinnvoll, um die Vielfalt sozialgeographischer Stimmen überhaupt hör- und sichtbar werden zu lassen. Erfolgt dieses könnte man in den Unterschieden vielleicht beides entdecken: Ähnliche Muster, die eher den universell gültigen Momenten zuzurechnen wären und die wegen der Dominanz der marktorientierten, neoliberalen Globalisierung voraussichtlich eher zu- denn abgenommen haben, und tiefgreifende Differenzen, die gleichwohl produktiv beispielsweise im interkulturellen Dialog und auf Basis gleichwertiger Sprecherpositionen ausgetauscht werden. Gewinnbringend wären weiterhin die Rekonstruktion sozialgeographischer Denkfiguren ausgehend von postmodernen Herausforderungen und die Identifizierung ihrer machtkritischen Gehalte. Im Vordergrund stehen damit Fragen der Emanzipation, Repression und Exklusion, die nicht nur in ihren gesellschaftlichen Kontexten transparent gemacht werden müssen, sondern auch für die Rechtfertigung von Wissenschaft bedeutsam sind. Vereinfacht ausgedrückt sollten die Grundlagen kritischer Sozialgeographie neu bestimmt werden, die trotz aller wichtigen Entwürfe, die unter der Bezeichnung Sozialgeographie auch im deutschsprachigen Raum zu finden sind, nicht hinreichend ausgearbeitet sind.

Neben solchen eher gesellschafts- und wissenschaftstheoretischen Aufgaben sollte auch die sozialgeographische Untersuchung des Rechtspopulismus und seiner Erfolgsmechanismen verstärkt werden. Das Postfaktische ist als normale, populäre Ausdrucksform anzusehen, die uns vielleicht nicht angenehm ist, aber die wissenschaftlich durchdrungen werden muss. Dazu gehört die Thematisierung der sozialräumlichen Polarisierung und der damit verbundenen Identitätspolitiken, die im Stichwort einer Sozialgeographie der Modernisierungsverlierer zusammengefasst werden könnte. Für solche Vorgehensweisen gibt es anregende Überlegungen wie das Konzept des Heartland (Jann, 2017) oder die amerikanische Diskussion über die gesellschaftspolitische Bedeutung des Stadt-Land-Gegensatzes (Cramer, 2016). In der aktuellen Situation könnte auch eine Überlegung weiterführend sein, die bereits Dubiel vor dem Hintergrund der damaligen historischen Situation im Übergang zum Postfordismus angeboten hat. Zum einen diskutiert er das ,populistische Moment" und damit die Frage, warum und unter welchen gesellschaftlichen Bedingungen der Populismus besonders bedeutsam wird bzw. wann er lediglich eine wenig wahrge- 
nommene Begleitform des gesellschaftlichen und politischen Wandels darstellt. Auf die heutige Zeit übertragen müssten die Befunde zur postpolitischen oder simulativen Demokratie (Crouch, 2008; Blühdorn, 2013) einen gesellschaftspolitischen Zusammenhang bilden, der zeigt, wie ,die kollektiven Kränkungserfahrungen, die Statusängste und frustrierten Glückserwartungen der betroffenen Bevölkerungsgruppen aus den etablierten Diskursen und Legitimationsmustern gleichsam herausfallen“ (Dubiel, 1985:650). Da in einem populistischen Moment ein Kampf um Deutungshoheit und Hegemonie entsteht, besteht zum anderen die wissenschaftliche Aufgabe, die Formen herauszuarbeiten, mit denen sich politische Akteure auf die herausgefallenen Milieus beziehen. Beispielsweise ruft die gegenwärtige politische Diskussion über Heimat geradezu nach entsprechender sozialgeographischer Einordnung.

Letztlich geht es um eine Debatte über wissenschaftliche Praxisformen, die im Sinne der Emanzipation und Befreiung sowie der Verhinderung von Repression wirken. Auch wenn wir dafür keine übergreifende Theorie mehr aufrufen können oder wollen, hat Wissenschaft eben nicht nur mit wissenschaftlich anerkannten Wissensbegriffen zu tun, sondern auch mit humanistischen Werten und Praktiken. Es ist gerade wieder einmal ein Zeitpunkt gekommen, entsprechende Haltungen deutlich zum Ausdruck zu bringen.

Datenverfüg barkeit. Für diesen Artikel wurden keine Datensätze genutzt.

Interessenkonflikt. Die Autoren erklären, dass kein Interessenkonflikt besteht.

Danksagung. Der Autor dankt den Gutachtern und dem Herausgeber für wertvolle und anregende Kommentare zu früheren Versionen dieses Beitrags. Vielen Dank auch an Jonathan Everts und Simon Runkel für ihr Engagement, das Thema Geographien sozialer Krisen/Krisen sozialer Geographie im Fach zu verankern.

Edited by: Benedikt Korf

Reviewed by: three anonymous referees

\section{Literatur}

Amin, A. (Hrsg.): Post-Fordism: a reader, Blackwell, Oxford, 1994. Beck, U.: Risikogesellschaft: Auf dem Weg in eine andere Moderne, Suhrkamp, Frankfurt am Main, 1986.

Becker, J.: Geographie in der Postmoderne? Zur Kritik postmodernen Denkens in Stadtforschung und Geographie, Institut für Geographie der Universität Potsdam, Potsdam, 1996.

Belina, B.: Germany in times of crisis: passive revolution, struggle over hegemony and new nationalism, Geograf. Ann. B, 95, 275$285,2013$.
Berger, J., Müller, J., und Pfriem, R. (Hrsg.): Materialienband zum Kongress Zukunft der Arbeit. Wege aus der Massenarbeitslosigkeit und Umweltzerstörung, Bielefeld, 1982.

Berman, S.: The Specter Haunting Europe: The Lost Left, J. Democracy, 27, 69-76, 2016.

Bieling, U. A.: Promotionskolleg - Rechtspopulistische Sozialpolitik und exkludierende Solidarität, Universität Tübingen, Tübingen, 2018.

Blühdorn, I.: Simulative Demokratie. Neue Politik nach der postdemokratischen Wende, Suhrkamp, Berlin, 2013.

Boltansky, L. und Chiapello, E.: Der neue Geist des Kapitalismus, UVK, Konstanz, 2003.

Bürk, T.: Antifaschismus, in: Handbuch Kritische Stadtgeographie, 3. Auflage, Herausgeber: Belina, B., Naumann, M., und Strüver, A., Westfälisches Dampfboot, Münster, 361-366, 2018.

Cramer, K. J.: The Politics of Resentment: Rural Consciousness in Wisconsin and the Rise of Scott Walker, University of Chicago Press, Chicago, 2016.

Crouch, C.: Postdemokratie, Suhrkamp, Frankfurt am Main, 2008.

Demirovic, A., Dück, J., Becker, F., and Bader, P. (Hrsg.): Vielfachkrise im finanzmarktdominierten Kapitalismus, VSA, Hamburg, 2011.

Dubiel, H.: Das Gespenst des Populismus, Merkur, 39, 639-651, 1985.

Franz, C., Fratzscher, M., und Kritikos, A. S.: AfD in dünn besiedelten Räumen mit Überalterungsproblemen stärker, DIW Wochenbericht 8/2018, 2018.

Fraser, N.: Vom Regen des progressiven Neoliberalismus in die Traufe des reaktionären Populismus, in: Die große Regression. Die internationale Debatte über die geistige Situation der Zeit, Herausgeber: Geiselberger, H., Suhrkamp, Berlin, 77-91, 2017.

Geiselberger, H. (Hrsg.): Die große Regression. Die internationale Debatte über die geistige Situation der Zeit, Suhrkamp, Berlin, 2017.

Grumke, T.: Sozialismus ist braun: Rechtsextremismus, die soziale Frage und Globalisierungskritik, in: Strategien der extremen Rechten. Hintergründe - Analysen - Antworten, Herausgeber: Braun, S., Geisler, A., und Gerster, M., Springer VS, Wiesbaden, 141-159, 2016.

Harvey, D.: The Condition of Postmodernity. An Enquiry into the Origins of Cultural Change, Blackwell, Oxford, 1989.

Hasse, J.: Die räumliche Vergesellschaftung des Menschen in der Postmoderne, Karlsruher Manuskripte zur Mathematischen und Theoretischen Wirtschafts- und Sozialgeographie, Geographisches Institut der Universität Karlsruhe, Karlsruhe, 91 pp., 1988.

Heisterhagen, N.: Kritik der Postmoderne. Warum der Relativismus nicht das letzte Wort hat, Springer VS, Wiesbaden, 2018.

Hillje, J.: Rückkehr zu den politisch Verlassenen, in: Gespräche in rechtspopulistischen Hochburgen in Deutschland und Frankreich, Das progressive Zentrum, Berlin, 2018.

Holtmann, E.: Völkische Fremdbilder. Ursprünge und Erscheinungsformen des Rechtspopulismus in Deutschland, Bpb Schriftenreihe 10207, Bundeszentrale für Politische Bildung, Bonn, 2018.

Jann, O.: „Heartland“ oder: Die Kritik der infamen Bürger, Sonderband 32, Leviathan, 45, 279-302, 2017.

Jensen, S. Q.: Othering, identity formation and agency, Qual. Stud., 2, 63-78, 2011.

Jensen, U.: Zornpolitik, Suhrkamp, Berlin, 2017. 
Jörke, D. and Nachtwey, O.: Das Volk gegen die (liberale) Demokratie? Die Krise der Repräsentation und neue populistische Herausforderungen, Sonderband 32, Leviathan, 45, 7-17, 2017.

Koppetsch, C.: Rechtspopulismus, Etablierte und Außenseiter. Emotionale Dynamiken sozialer Deklassierung, Sonderband 32, Leviathan, 45, 208-232, 2017.

Koppetsch, C.: Viele Linke machen sich etwas vor, Taz vom 7 Juli 2018 .

Koschorke, A.: Die akademische Linke hat sich selbst dekonstruiert. Es ist Zeit, die Begriffe neu zu justieren, NZZ vom 18 April 2018.

Kreutzmann, H. and Reuber, P.: Kulturerdteile im Wandel? Politische Konflikte und der clash of civilizations, in: Geographie heute - für die Welt von morgen, Herausgeber: Ehlers, E. und Leser, H., Klett-Perthes, Gotha, Stuttgart, 139-146, 2002.

Krüger, R.: Die Geographie auf der Reise in die Postmoderne? Wahrnehmungsgeographische Studien zur Regionalentwicklung, BIS Verlag, Oldenburg, 1988.

Lossau, J.: Kulturgeographie als Perspektive. Zur Debatte um den cultural turn in der Humangeographie - eine Zwischenbilanz, Berichte zur deutschen Landeskunde, 82, 317-334, 2008.

Lyotard, J.-F.: Das postmoderne Wisse, Ein Bericht, Bremen, 1982.

Marchart, O.: Das unmögliche Objekt. Eine postfundamentalistische Theorie der Gesellschaft, Suhrkamp, Berlin, 2013.

Merkel, W.: Kosmopolitismus versus Kommunitarismus. Ein neuer Konflikt in der Demokratie, in: Parties, Governments and Elites: The Comparative Study of Democracy, Herausgeber: Harfst, P., Kubbe, I., and Poguntke, T., Springer VS, Wiesbaden, 9-24, 2017.

Mudde, C. and Rovira Kaltwasser, C.: Populism: A very short introduction, Oxford University Press, Oxford, 2017.

Mudde, C. and Rovira Kaltwasser, C.: Studying Populism in Comparative Perspective: Reflections on the Contemporary and Future Research Agenda, Comparat. Polit. Stud., 51, 1667-1693, 2018.
Müller, J.-W.: Was ist Populismus? Ein Essay, Suhrkamp, Berlin, 2016.

Nachtwey, O.: Die Abstiegsgesellschaft. Über das Aufbegehren in der regressiven Moderne, Suhrkamp, Berlin, 2016.

Oßenbrügge, J.: Territorial Ideologies in West Germany 19451985: Between nationalist geopolitics and regionalist attitudes, Polit. Geogr. Quart., 8, 387-399, 1989.

Paoli, G.: Die lange Nacht der Metamorphose. Über die Gentrifizierung der Kultur, Matthes und Seitz, Berlin, 2017.

Peet, R. and Hartwick, E.: Theories of Development, in: Contentions, Arguments, Alternatives, 3rd Edn., Guilford, New York, 2015.

Pollack, D.: Modernisierungstheorie - revised: Entwurf einer Theorie moderner Gesellschaften, Z. Soziolog., 45, 219-240, 2016.

Priester, K.: Populismus. Historische und aktuelle Erscheinungsformen, Campus, Frankfurt am Main, 2007.

Sedlacek, P. (Hrsg.): Kultur/Sozialgeographie, UTB Schöningh, Paderborn, 1982.

Steiner, N. D. and Landwehr, C.: Populistische Demokratiekonzeptionen und die Wahl der AfD: Evidenz aus einer Panelstudie, Politische Vierteljahresschrift, 59, 463-491, 2018.

Streeck, W.: The Return of the Repressed. New Left Review 2017 (104), dt. Fassung: Die Wiederkehr der Verdrängten als Anfang vom Ende des neoliberalen Kapitalismus, in: Die große Regression. Die internationale Debatte über die geistige Situation der Zeit, Herausgeber: Geiselberger, H., Suhrkamp, Berlin, 253-274, 2017.

Wodak, R.: Vom Rand in die Mitte - ,schamlose Normalisierung“, Politische Vierteljahresschrift, 59, 323-335, 2018. 